\title{
EDUCACIÓN Y TRABAJO DE LOS NIÑOS COMO REALIDADES CONEXAS. LA EDUCACIÓN OBLIGATORIA COMO DERECHO A SER EJERCITADO PREFERENTEMENTE POR LOS MENORES.
}

\author{
EDUCATION AND WORK OF CHILDREN AS RELATED FACTS. \\ COMPULSORY EDUCATION AS A RIGHT TO BE EXERCISED \\ PREFERABLY BY MINORS.
}

Amaya Suárez Malaxechevarría ${ }^{1}$

RESUMEN -La crisis económica que atravesamos implica también crisis social, desde el momento en el que los recortes a que nos vemos sometidos recaen fundamentalmente en derechos sociales, justificándose aquéllos en una continuidad del sistema económico. Uno de los derechos sociales por excelencia, la educación, como camino con una trayectoria diferente según los casos, resulta fundamental y obligado para el desarrollo de la persona. Su histórica vulneración sufrida por los menores, y su innegable relación con el mundo del trabajo, es el objetivo del presente artículo.

PALABRAS CLAVE - Educación. Trabajo. Niños.

ABSTRACT - The economic crisis we are facing social crisis also means, from the time the cuts we are subjected to rest primarily with social rights, justifying a continuation of those in the economic system. One of the quintessential social rights, education as career path with different vessels, is essential and required for the development of the individual. His historic breach experienced by children, and their undeniable relationship to the world of work is the objective of this article.

KEYWORDS - Education. Work. Children.

\section{PLANTEAMIENTO.}

El derecho a la educación es uno de los pilares para la abolición del trabajo infantil por lo que su plena efectividad y desarrollo es obligación de

\footnotetext{
${ }^{1}$ Doctora en Derecho por la Universidad de Burgos. Tesis doctoral: "Los derechos de los niños en el mundo del trabajo" (2011) dentro del Programa de Doctorado de Derecho Público de la UBU: "Sociedad Plural y Nuevos Retos del Derecho", Área de Filosofía del Derecho. E-mail: amaiasuma@gmail.com.
} 
todos los Estados. A esto se suma su obligatoriedad y gratuidad, nueva muestra de la exigencia de la intervención del Estado en el marco social y democrático de Derecho. $Y$ es que la escolarización y educación han de ser obligatorias y gratuitas para garantizar el desarrollo personal y social.

No obstante lo anterior, la educación no ha sido siempre respetada, y han sido demasiadas las muestras históricas que determinaron su instrumentalización al servicio de intereses y beneficios puramente económicos, explotándose a los niños, y vulnerándose sus derechos más básicos, como así lo han demostrado innumerables estudios y visiones. A pesar de esto, hoy en día existen millones de niños trabajadores, y en no pocos casos en tareas especialmente peligrosas. ${ }^{2}$

\section{LA EDUCACIÓN Y SU RELACIÓN HISTÓRICA CON EL TRABAJO DE LOS NIÑOS.}

Decimos que la educación es uno de los derechos cuyo ejercicio normalizado es imprescindible para la erradicación del trabajo y explotación infantil, puesto que afecta no sólo a la vida de cada niño, sino también a la sociedad en la que se encuentra inserto, es decir, a su progreso.

Este es el motivo por el que el estudio de la educación tiene relevancia, especialmente por la importancia que ha merecido y que merece en orden a la fundamentación de la prohibición del trabajo de los niños, ya que trabajo y educación son "dos caras de la misma moneda." Lo que se va a procurar a continuación será mostrar la relación evidente que ha existido y existe entre ambas.

A pesar de la imposibilidad de un análisis histórico exhaustivo de la educación como realidad y como derecho, sí que abordamos unos momentos históricos concretos y algunas posturas relevantes que confirman la primera

\footnotetext{
${ }^{2}$ La más reciente estimación mundial de la Organización Internacional del Trabajo, realizada en 2011, revela que 115 millones de niños están involucrados en trabajo peligroso. Se trata de trabajos, que por su naturaleza o por las condiciones en que se lleva a cabo, es probable que dañe la salud, la seguridad 0 la moralidad de los niños. http://www.ilo.org./ipec/Informationresources.
} 
afirmación, unas enfocadas al acceso a la educación, y otras a su propio contenido.

\subsection{La educación en la Antigüedad como instrumento sobre el que formar futuros ciudadanos.}

Hemos comenzado con la afirmación de que educación y trabajo de los niños no son realidades opuestas o alejadas, sino todo lo contrario, mostrándose como parámetros para poder analizarse una y otro. En la Antigüedad, la educación de los niños era algo fundamental para la búsqueda de ciudadanos virtuosos en una sociedad ideal, pero era el logro de este tipo de sociedad el interés primordial, no existiendo ningún otro dirigido a la formación y desarrollo del menor en su consideración de tal. ${ }^{3}$ La búsqueda de este ciudadano virtuoso es la razón del establecimiento de una educación encaminada en tal sentido, considerándose al niño como objeto, más que como sujeto, titular de algún interés por sí mismo. Esta es la visión que se encuentra en Platón y Aristóteles para quienes - y en línea con los valores imperantes en sus sociedades - los niños carecían de valor por sí mismos. ${ }^{4}$

Esta apreciación del niño como objeto se acredita fundamentalmente por el hecho de que eran un valor para los padres y sólo en este valor se centraba su interés. La prioridad para los padres no era la educación de sus hijos, sino la posibilidad de ser utilizados en el ámbito familiar para la realización de tareas o trabajos; la instrucción de los menores era algo subsidiario, y - desde luego distaba mucho de asemejarse a un derecho sino que se consideraba un

\footnotetext{
${ }^{3}$ Como recuerda CAMPOY: "Los niños constituyen la materia - el mármol - de mejor o peor naturaleza - de mejor o peor calidad -, con la que el legislador - escultor -, ha de formar esculpir -, mediante el buen uso de la educación - del cincel -, el más perfecto ciudadano - la más perfecta escultura. La educación no era importante para el interés del propio menor, sino para la formación de futuros ciudadanos virtuosos, teniendo los niños la consideración de materia sobre la que desplegar la formación que sea más adecuada para el logro de la sociedad ideal', CAMPOY CERVERA, I., "La negación de los derechos de los niños en Platón y Aristóteles", Cuadernos Bartolomé de las Casas, Dykinson, 2006, op. cit., p. 28.

${ }^{4}$ Por tanto, no existía infanticidio o abandono de niños. El motivo de su práctica lo destaca CAMPOY en "la ausencia de estar perjudicando algo valioso (...) el colectivo más perjudicado por ello fuesen los niños que sus deficiencias psíquicas o físicas se considerase que no podrían llegar jamás a convertirse en el futuro adulto pretendido por la sociedad o por sus padres.", CAMPOY CERVERA, I., "La negación de los derechos...", op. xit., p. 81.
} 
instrumento ideal para la construcción de un futuro adulto inserto en una sociedad virtuosa, a la vez que suponía un coste para los padres, ya que los niños podían ser usados para trabajar.

Por lo tanto, la educación en la Antigüedad no era un derecho de los niños, sino que era un recurso que raramente se utilizaba, ya que la consideración del niño como objeto merecía ser empleado en otras tareas y trabajos por sus propios padres, siendo plenamente admitido por la sociedad de entonces.

\subsection{La educación durante la Revolución Industrial. La educación como instrumento para rebajar costes.}

Otro hito que consideramos importante en materia de educación lo encontramos con el surgimiento y desarrollo de la Revolución Industrial de los Siglos XVIII y XIX, sin duda, una de las épocas de mayor crueldad en este terreno. No sólo estaba permitido sino que éste se fomentaba abiertamente formando parte de una realidad social en la que las grandes masas de trabajadores se convirtieron en sufridoras de los avances tecnológicos e industriales ${ }^{5}$. En este sentido, la natural consecuencia del establecimiento de las fábricas o industrias en las ciudades produjo que las familias que vivían en los ámbitos rurales se desplazaran a los centros urbanos para emplearse. La introducción de la máquina en el proceso productivo originó salarios muy bajos, largas jornadas laborales e incluso la utilización de la mano de obra infantil, porque los míseros jornales que se les daban no cubrían en absoluto la subsistencia de todos los miembros de la unidad familiar, lo cual provocaba que los más pequeños tuvieran que aportar a la unidad familiar, de una u otra manera.

El hecho de que mencionemos expresamente la Revolución Industrial como época en la que el trabajo y explotación infantil eran normales, no significa que con anterioridad no existiera, puesto que era común y admitido

\footnotetext{
${ }^{5}$ La máquina de vapor, la lanzadera volante y otros inventos de John Kay y Samuel Crompton, supusieron la transformación de la técnica y de la producción, junto a una reducción considerable de la mano de obra, lo que originó una primera reacción obrera hostil contra la propia máquina.
} 
que los niños ayudaran a sus familias, en pequeños talleres o, principalmente, en los trabajos agrícolas que constituían la forma productiva principal. Sin embargo, la diferencia radicaba en que en el desempeño de estos trabajos agrícolas el grado de esfuerzo de los niños estaba sometido a una concreta periodicidad, lo que les daba la oportunidad de acudir con una cierta frecuencia a la escuela. ${ }^{6}$ Esta posibilidad de acudir a la escuela con cierto aprovechamiento para los niños fue borrada por completo con la entrada en las fábricas de los más pequeños.

El surgimiento del proletariado supuso, también, un empeoramiento de las condiciones de vida con respecto a la realidad de los artesanos y campesinos preindustriales, que hacía necesaria la mano de obra infantil en talleres, fábricas y minas para cubrir las necesidades por el tan bajo salario que se recibía. Las condiciones deplorables en que se encontraban ocasionaron el surgimiento de la conciencia de clase y de la oposición del capital y el trabajo asalariado $^{7}$. Sin duda, ZOLA describió perfectamente estas realidades en su obra "Germinal" donde relata la huelga de los trabajadores de las cuencas mineras francesas en el siglo XIX y en las que niños y niñas eran unas piezas más en la extracción de materiales, sin distinción con los mayores, sometidos a las peores condiciones de vida y de trabajo. ${ }^{8}$

\footnotetext{
${ }^{6}$ Así, CIPOLLA describe lo que para él consistía una diferencia radical: "La diferencia - una diferencia sustancial por cierto - consistía no sólo en que el trabajo en el campo era más sano, sino también en que las faenas agrícolas se limitaban necesariamente a una o dos estaciones al año. En invierno, los hijos más pequeños de los campesinos no trabajaban y, por tanto, podían ser enviados a la escuela, con un opportunity cost nulo. De este hecho se derivaba otro, a saber, el carácter fluctuante de la asistencia a la escuela en la era preindustrial.", CIPOLLA, C., "Educación y desarrollo en occidente", Ariel, Barcelona, 1970, op. cit., p. 26.

7 En palabras de SANGUINETTI: "La expansión del proletariado y su concentración en las fábricas propiciaron el surgimiento en los trabajadores de la conciencia de la posibilidad de actuar colectivamente con el fin de remediar su situación. Surgieron, así, las primeras acciones reivindicativas, inicialmente articuladas alrededor de las coaliciones obreras y más tarde de los sindicatos, a través de las cuales los obreros buscaron oponer al poder del empresario y la inhibición del Estado la defensa conjunta de sus intereses.", SANGUINETTI RAYMOND, W., "EI derecho del trabajo como categoría histórica", en V.V.A.A., "Mercado y organización del trabajo en España (Siglos XIX y XX)", Grupo Editorial Atril 97, Sevilla, 1998, op. cit., p. 467.

${ }^{8}$ ZOLÁ narra y describe el contraste existente entre las condiciones de los trabajadores que viven en los poblados cercanos a las minas - personajes como Étienne, Mauheu, Catherine, Chaval, Jeanline, y muchos otros - y los dueños de estas últimas (Montsou, Voureaux), con conflictos y sumisiones entre ellos. La huelga iniciada como consecuencia de una rebaja del salario y de las condiciones del trabajo origina sufrimiento y muerte que se ceba con quienes menos tienen y que, irremediablemente, han de volver a la mina: " $Y$ los compañeros seguían golpeando, cada vez con más claridad, como si se hubieran acercado al suelo. Bajo los rayos encendidos del astro, en aquella mañana de juventud la campiña estaba preñada de aquel
} 
Este cambio del sistema productivo y económico trajo consecuencias sociales: pobreza, pillajes y robos - también por los más pequeños, que había que esconder y, para ello, se empleaban "escuelas" como un método o forma de llevarlo a cabo. ${ }^{9}$ Por lo tanto, el qué hacer con los menores fue resuelto de forma productiva. Una de las fórmulas fue la de "enseñanza de un oficio", en la que bajo la fórmula del "aprendiz" se escondía una auténtica explotación infantil durante interminables horas de trabajo y condiciones penosas. ${ }^{10}$

Las escuelas de beneficencia, las enseñanzas de trabajo y la educación no eran garantías o derechos de los niños - los cuales no eran titulares ni ejercían derecho alguno - sino que eran medidas o instrumentos tendentes a hacer efectivo un "derecho a no percibir la pobreza y sus consecuencias ". Por lo tanto, este trabajo infantil estaba perfectamente determinado e incluso fomentado en la Europa liberal del Siglo XIX, considerándose a los niños como una auténtica fuerza productiva, capaz de generar enormes rendimientos con un coste escasísimo. Por eso, no sólo se trataba de borrar u ocultar las tremendas huellas sociales que sufría la población, sino de aprovecharlas para sacar el máximo rendimiento económico. El trabajo infantil era "vendido" como un beneficio para todos, no sólo para el propio empresario, sino también para el propio niño que en el futuro gozaría de la mayor de las destrezas para continuar siendo un gran productor. ${ }^{11}$

rumor. Los hombres empujaban, un ejército negro, vengador, que germinaba lentamente en los surcos, creciendo para las cosechas del siglo futuro, cuya germinación pronto hará estallar la tierra.", ZOLÁ, E., "Germinal", Austral, Madrid, 2008, op. cit., p. 507.

${ }^{9}$ CUNNINGHAM recuerda que: "Se utilizaban las técnicas del trabajo y la escolarización sólo para evitar la mendicidad en las calles. Eran los "hijos de los pobres", CUNNINGHAM, H, "Trabajo y explotación infantil: situación en la Inglaterra de los siglos XVII al XX", Ministerio de Trabajo y Seguridad Social, Madrid, 1994, op. cit., p. 14.

${ }_{10}$ GORDON recuerda la existencia en Londres de los llamados "Overseer of the Poor" o autoridades encargadas de los pobres que: "(...) para aliviar el peso cada vez mayor que suponía costear la subsistencia de los pobres con los impuestos locales, ofrecían lotes de niños de los hospitales a las fábricas del norte. Estos aprendices eran entregados a sus empleadores a partir de los siete años de edad, y vivían en "casas de aprendices", contiguas a la fábrica. Además de las condiciones de vida con frecuencia miserables que tenían que soportar, trabajaban desde las 5 de la mañana hasta las 8 de la tarde, con pauses de media hora para el desayuno y el almuerzo.", GORDON, P., "Robert Owen (1771-1858)", Perspectivas: revista trimestral de educación comparada (París, UNESCO: Oficina Internacional de Educación), vol. XXIV, no 1-2, 1993, pp. 279-297. http://www.ibe.unesco.org/fileadmin/user_upload/archive/publicarion/ThinkersPdf/owens.pdf.

${ }_{11}$ CARRO recuerda las manifestaciones del político de la época William Pitt al respecto: “(...) la experiencia ha demostrado todo lo que puede producir el trabajo de los niños y la ventaja que hay en emplearles desde temprana edad en trabajos que son capaces de realizar. El 


\subsection{Ideas Liberadoras. Rousseau y Fröbel. La educación como proceso natural.}

Las ideas anteriores contrastaron con las de otros pensadores y autores, para quienes la educación era considerada como un exponente, como motor de la formación de las personas, con un valor como tal. Sin duda, un cambio en la concepción de la educación lo constituyeron obras como las de ROUSSEAU, porque su obra "El Emilio" supuso un auténtico nuevo tratado sobre la infancia y la educación de los niños, respetuoso con su libertad y dignidad. ${ }^{12}$

En la misma línea nos encontramos con el autor alemán FRÖBEL, de cuya lectura se desprenden los mismos principios de autonomía del niño, el cual necesita del espacio de libertad adecuado para alcanzar su propio desarrollo en toda su extensión. ${ }^{13}$ La correcta educación que el niño ha de recibir, se encuentra en su "propia reflexión" en la que se incluye la asunción de tareas o responsabilidades de forma natural.

Para este autor, el niño es visto en un concepto mucho más amplio que el de merecedor de protección sino que la visión es más integradora o global en la sociedad. A este respecto afirma: "...lo primero y más importante de la educación de los niños es despertar pronto en ellos la propia reflexión. Y no hablaba de despertar también su actividad y amor al trabajo, porque esto le parecería tan natural que ni siquiera hacía falta comunicarlo. Por lo demás un niño en quien se ha desarrollado la reflexión, ¿no está ya favorablemente

desarrollo de las Escuelas de industrial también debe dar resultandos materiales importantes. $\mathrm{Si}$ alguien se molestar en calcular el valor total de lo que ganan desde ahora los niños educados según este método quedaría sorprendido al ver de qué carga, con su trabajo, bastante para proveer a su mantenimiento, alivian al país, y el complemento que sus esfuerzos laboriosos y las costumbres en las que se forman aportan a la riqueza nacional", CARRO IGELMO, A.J., "Historia social del Trabajo", Bosch, Barcelona, 1985, op. cit., p. 289.

${ }^{12}$ Vid. ROUSSEAU, J.J., "El Emilio o de la Educación", Edaf, Madrid, 1977.

${ }^{13}$ Afirma FRÖBEL: "Por lo general, tratándose de plantas $O$ animales, nos contentamos con darles espacio y tiempo, no ignorando que, según las leyes que rigen cada especie, habrán de crecer bien y adquirirán hermoso desarrollo. A plantes y animales se les deja en paz, evitando cualquier influencia perturbadora, que sólo serviría para impedir su libre y sano desenvolvimiento. Pero, en cambio, el hombre en su niñez parece ser para el hombre un pedazo de cera, una masa de arcilla con la que pueda modelarse lo que se quiera.", FRÖBEL, F., "El respeto a la libertad de la naturaleza", en "La educación del hombre", en V.V.A.A., "La educación en los tiempos modernos. Tiempos y documentos". Dir. Julio Ruiz Berrio, Actas, Madrid, 1996, op. cit., p. 143.

REVISTA DO DIREITO UNISC, SANTA CRUZ DO SUL No 37|p. 161-178| JAN-JUN 2012 
dispuesto para la laboriosidad y para todas las virtudes domésticas y sociales?" ${ }^{14}$ Combina este autor factores como la libertad y la laboriosidad, de forma que para llegar a la segunda ha de haberse pasado previamente por ese tiempo de libertad y de respeto que implica el conocimiento de sí mismo y de su entorno, objeto mismo de la educación.

\subsection{La educación en Marx. La educación como medio para conseguir fuerza social.}

La relación entre educación y trabajo también fue plasmada por MARX quien puso de manifiesto la existencia de la explotación de los niños por el sistema capitalista, por la realidad histórica de la explotación de los niños en las fábricas, bajo el lema "quien no trabaja no come"15. Afirmó: "Cuanto mayor es el desarrollo de la industria moderna, mayor es la proporción en que el trabajo de los hombres es suplantado por el de las mujeres y los niños. Las diferencias de edad y sexo pierden toda significación social para la clase obrera"16. No obstante lo anterior, él mismo llegó a admitirlo según distintas franjas de edades y a criterios de orden fisiológico ${ }^{17}$, resultando fundamental para transformar todo el modo de producción la educación pública y gratuita de todos los niños. ${ }^{18}$ Por educación, entendía Marx educación mental, educación corporal - producida por ejercicios gimnásticos y militares - y educación

\footnotetext{
${ }^{14}$ FRÖBEL, F., "El respeto...", op. cit., p. 145.

${ }^{15}$ MARX afirmó: "Consideramos la tendencia de la industria moderna a hacer cooperar los niños y jóvenes de ambos sexos en el gran movimiento de la producción social como un progreso y una tendencia legítima, aunque el modo en que esta tendencia se realiza bajo el yugo del capital, sea una abominación.", MARX, K., "Trabajo de jóvenes y niños de ambos sexos", "Instrucciones a los delegados del Consejo Central Provisional", en "La educación en los tiempos modernos. Tiempos y documentos", Director Julio Ruiz Berrio, Actas, Madrid, 1996, op. cit., p. 156.

${ }^{16}$ MARX, K., "El Manifiesto Comunista", en MARX, K., y ENGELS, F., "Manifiesto Comunista y otros escritos políticos", Grijalbo, Barcelona, 1975, op. cit., p. 32.

${ }^{17}$ Así: "A causa de razones fisiológicas, juzgamos conveniente dividir los niños y jóvenes de ambos sexos en tres clases, que deben tratase diferenciadamente. La primera clase comprende los niños de 9 a 12 años, la segunda de 12 a 15, y la tercera de 15 a 18. Proponemos que el empleo del primer grupo, en cualquier trabajo, sea en fábricas o en casas particulares, sea legalmente restringido a dos horas, el del segundo a cuatro y el del tercero a seis. Para el tercer grupo, debe haber una interrupción de al menos una hora par la comida y la recreación.", MARX, K., "Trabajo de jóvenes y niños de ambos sexos. Instrucciones a los delegados del Consejo Central Provisional", en "La educación en los tiempos modernos. Tiempos y documentos", Dir. Julio Ruiz Berrio, Actas, Madrid, 1996, op. cit., p. 156.

${ }^{18}$ MARX, K., "El Manifiesto...", op. cit., p. 49.
} 
tecnológica - abrazando los principios generales y científicos de cualquier producción y al mismo tiempo iniciando a los niños y jóvenes en el manejo de instrumentos elementales en toda industria- ${ }^{19}$ En este sentido, toma partido, considerando necesaria la educación no sólo para la formación de la persona, sino principalmente para evitar lo que llama una abominación, el permitir que niños y niñas trabajen en las fábricas en condiciones realmente penosas, siendo un instrumento más, un engranaje más en el sistema productivo. ${ }^{20} \mathrm{~A}$ su vez, la considera necesaria, como hemos visto, para que se aprenda el manejo de los más básicos instrumentos de toda industria.

En segundo lugar, se observa que establece una franja de edades por las que se va permitiendo la entrada de los menores en el mundo del trabajo. Consecuencia natural de lo anterior, es decir, el hecho de que se establezca la posibilidad de incorporarse al trabajo por parte de los ya denominados niños y jóvenes trae como consecuencia que tiene que estar necesariamente subordinado o complementado con una formación. ${ }^{21}$

Admite el trabajo de los menores, pero siempre y cuando sea compatible con la necesaria educación que merecen para que la razón social pueda convertirse en fuerza social. Así, el estudio de la educación es básico desde el momento en el que se toma como primer parámetro y uno de los principales fundamentos para la actividad de los menores.

\section{5.- La educación como elemento para la perpetuación de las estructuras de poder.}

\footnotetext{
${ }^{19}$ MARX, K.,"Trabajo de jóvenes...", op. cit., p. 157.

${ }^{20}$ Afirmó: "La sociedad no puede permitir, ni a padres ni a patronos, emplear para el trabajo niños y jóvenes, a menos de combinar este trabajo productivo con la educación."MARX, K., "Trabajo de jóvenes...", op. cit., p. 157.

${ }^{21}$ Así: " $A$ la división de niños y jóvenes en tres grados de 9 a 18 años, debe corresponder una graduada y progresiva educación mental, gimnástica y tecnológica. Exceptuando tal vez el primer grupo, la manutención de estas escuelas politécnicas debe en parte cubrirse con la venta de sus propios productos. Esta combinación de trabajo productivo pagado con educación mental, ejercicios corporales y aprendizaje tecnológico, llevará a las cases obreras muy por encima del nivel de las clases burguesas y aristocráticas. Se sobreentiende que el empleo de cualquier persona de 9 a 18 años en un trabajo nocturno o en cualquier industria de efectos nocivos a la salud, debe estar severamente prohibido por la ley."MARX, K.," Trabajo de jóvenes...", op. cit., p. 157.
} 
Más modernamente, son diversos los autores que han considerado la educación no ya como un instrumento relacionado con el sistema productivo, sino como un instrumento para la perpetuación de las relaciones de poder, de forma que tal que la libertad del educando en su proceso de asunción de conocimientos queda bastante limitada por las estructuras creadas en el sistema educativo, consideradas éstas siervas de quienes detentan el poder. No se analiza tanto el problema del acceso a la educación sino en cómo es ésta, sus finalidades.

Autores como CIRIGLIANO manifiestan que la educación se presenta como un instrumento de relación entre el hombre y la sociedad enfrentados. ${ }^{22}$ Al igual que ocurre en las estructuras tribales o primitivas ${ }^{23}$, la sociedad requiere que los individuos estén preparados para poder perpetuarla. ${ }^{24} \mathrm{El}$ impartir los ingredientes culturales propios y su asimilación por parte de los individuos es la educación, un instrumento no tanto al servicio del individuo, sino al servicio de la sociedad, del grupo. ${ }^{25}$ Así, familia, Iglesia o Estado son estructuras sociales o grupos que tienen fines sociales particulares, afectando a la educación, que es diferente dependiendo del grupo al que se pertenezca. Para este autor el Estado, como un grupo más - el más poderoso - transmite su propia educación: "Se suele encargar al Estado esta tarea común por ser el organismo más poderoso (no en vano es el del Poder), pero, como han hecho con él, los círculos sociales (teóricamente) pueden elegir a cualquier otro poderoso (Iglesia en medioevo) y confiarle lo común y básico para todos”26

Otros autores como FREIRE o APPLE exponen abiertamente la relación de la educación con la opresión y con la permanencia de las estructuras de

${ }^{22}$ CIRIGLIANO, G., F., "Filosofía de la educación"”, Humanitas, Buenos Aires, 1972, op. cit., p. 12.

${ }^{23}$ CIRIGLIANO, G., F., "Filosofía...", op. cit., p. 14.

24 Para CIRIGLIANO: "La caracterización de socios sólo la obtienen los individuos cuando logran asimilar (poseer y utilizar como propios) los elementos culturales reinantes en la sociedad", CIRIGLIANO, G., F., “Filosofía...”, op. cit., p. 14.

${ }^{25}$ La educación está al servicio de la sociedad, entendiendo por ésta, los grupos que la constituyen: "...y ella no parece ser más que la unión o reunión orgánica de todos ellos. Nos hallamos ahora frente a lo que se llama sociedad como distinta de comunidad. En la antigüedad, la comunidad (sin diferencia de grupos) educaba al par que vivía comunitariamente. Es a partir de la división del trabajo que obtenemos grupos y por tanto sociedad, como la que ahora hallamos.", CIRIGLIANO, G., F., "Filosofía...", op. cit., p. 21.

${ }^{26}$ Manifiesta este autor: "Una vez producida la unificación de la educación, ésta se convierte en un arma, un medio por el que se controla toda la sociedad, se la guía, maneja y cambia", CIRIGLIANO, G., F., "Filosofía...”, op. cit., p. 29. 
poder, respectivamente. ${ }^{27}$ En este sentido, FREIRE parte de la existencia de una relación entre educación y opresión. La opresión implica una falta de libertad; esto es claro. El opresor obstaculiza la búsqueda de la libertad. Late en la opresión una falta de ejercicio de la libertad que genera un miedo a lo desconocido, que genera que la propia falta de libertad se vea como seguridad que sienten los oprimidos. ${ }^{28}$ De manera que el opresor genera seguridad en quien lo sufre, de tal modo, que es preferible estar oprimido y sin conciencia que el uso propio de la libertad, lo que genera una relación de dependencia entre opresores y oprimidos, que se presenta como una de las causas de la perpetuación de la opresión ${ }^{29}$

FREIRE propone desechar la pedagogía bancaria, en la que el educador es un mero narrador que va rellanando de contenidos a los educandos, considerados éstos como meros depósitos. ${ }^{30}$ En contraposición a este tipo de

${ }^{27}$ SUBIRATS destaca igualmente el caso de la reciente historia de España en la que la influencia de la política en la educación ha sido clara: la educación como modo de perpetuación de los grupos sociales, SUBIRATS, M., "Sociología de la Educación" en V.V.A.A. "Sociología en España", Comp. Salvador Giner y Luis Moreno, Consejo Superior de Investigaciones Científicas", Madrid, 1990, op. cit., p. 168.

${ }^{28}$ Califica a los oprimidos como: "acomodados y adaptados, inmersos en el propio engranaje de la estructura de dominación que temen a la libertad, en cuanto no se sienten capaces de correr el riesgo de asumirla. La temen también la medida en que luchar por ella significa una amenaza, no sólo para aquellos que la usan, para oprimir esgrimiéndose como sus "propietarios" exclusivos, sino para los compañeros oprimidos, que se atemorizan con mayores represiones."FREIRE, P, "Pedagogía del oprimido", Siglo Veintinuno de España Editores, Madrid, 2000, op. cit., p.44. Continúa este autor: "Los oprimidos, que introyectando la "sombra" de los opresores siguen sus pautas, temen a la libertad en la medida en que ésta, implicando la expulsión de la "sombra", exigiría de ellos que "llenaran" el "vacío" dejado por la expulsión, con "contenido" diferente: el de su autonomía. El de su responsabilidad sin la cual no serían libres. La libertad, que es una conquista y no una donación, exige una búsqueda permanente. Búsqueda que sólo existe en el acto responsable de quien la lleva a cabo. Nadie tiene libertad para ser libre, sino que, al no ser libre lucha por conseguir su libertad. Esta tampoco es un punto ideal fuera de los hombres, al cual inclusive, se alienan." FREIRE, P, "Pedagogía...", op. cit., p.47.

${ }^{29}$ Explica FREIRE: "El miedo a la libertad, del que, necesariamente no tiene conciencia quien lo padece, lo lleva a ver lo que no existe. En el fondo, quien teme a la libertad se refugia en la "seguridad vital", para usar la expresión de Hegel, prefiriéndola a la "libertad arriesgada" (...) "Solamente arriesgando la vida se mantiene la libertad...El individuo que no ha arriesgado la vida puede sin duda ser reconocido como persona que no ha alcanzado la verdad de este reconocimiento como autoconciencia independiente", Fenomenología del Espíritu - Fondo de Cultura - México, 1966, p. 116, vid. en FREIRE, P, “Pedagogía...", op. cit., pp. 28 y 29.

${ }^{30}$ Existe una educación bancaria o tradicional, en virtud de la cual, lo que se está es promoviendo el status quo, que no varíen las cosas para la persistencia de la opresión. Esta concepción bancaria de la misma implica que el educador es un mero narrador que va rellenando de contenidos a los educandos que son considerados como unos depósitos. De ahí que se denomine "educación bancaria". No existe relación entre educador y educando. Así: "(...) el único margen de acción que se ofrece a los educandos es el de recibir los depósitos, guardarlos y archivarlos. Marquen que sólo les permite ser coleccionistas o fichadotes de cosas 
educación expuesta propone un tipo de educación que busca la liberación, una superación de la anterior en la que tanto educando como educadores se encuentran no en situación de oposición, sino de dialéctica de la que ambos aprenden. Es la manera de lograr el cambio para eliminar la situación de opresión y lograr la libertad, la libertad de conciencias, la libertad de los hombres. $^{31}$

Pero este autor no es el único que relaciona de forma directa y abierta educación y poder/economía, siendo también representante de ello APPLE, quien afirma el importante papel de las escuelas en la reproducción de un orden social estratificado, que: "descansa sorprendentemente en la desigualdad por razón de sexo, clase o raza” ${ }^{32}$. Concreta la situación de los trabajadores y la situación de las mujeres y de minorías raciales de difíciles condiciones objetivas que tienen que afrontar. Lo explica APPLE en la rápida centralización y concentración de los recursos y el poder económico y cultural, definiéndolo como el "poder corporativo". ¿Cuál es el papel que desempeña la escuela en este ámbito?: "...la escuela genera espontáneamente ciertos tipos de discriminación (...) por un lado el papel de la escuela como aparato ideológico estatal en la creación de agentes (con las disposiciones y valores apropiados) para cubrir las necesidades de la división social del trabajo en la sociedad y por otro lado con el papel de las instituciones educativas en la producción de tipos de conocimiento concreto que precisa una sociedad desigual. ${ }^{33}$ Después de recalcar la diferencia entre las que denomina las dos grandes posturas en materia de educación - teorías del capital humano y de

que archivan." Para este autor la relación que debe existir entre educador y educando no ha de ser la de que el primero es el sabio y es el único que enseña, sino que también aprende con los educandos, FREIRE, P, "Pedagogía...", op. cit., p. 76.

${ }^{31}$ FREIRE describe este tipo de educación propuesta como: "comprometida con la liberación, se empeña en la desmitificación. Por ello, la primera - refiriéndose a la "bancaria" - niega el diálogo en tanto la segunda tiene en él la relación indispensable al acto cognoscente, desvelador de la realidad", FREIRE, P, "Pedagogía...", op. cit., p. 95.

${ }^{32}$ Continúa señalando que: "Personas tan diversas como Bourdieu, Althusser, Baudelot y Establet en Francia; Bernstein, Young, Whitty y Willis en Inglayerra; Kallos y Lundgren en Suecia; Gramsci en Italia; Bowles, Gintins, yo mismo y otros en Estados Unidos, hemos afirmado repetidamente que el sistema educativo y cultural es un elemento extraordinariamente importante para el mantenimiento de las actuales relaciones de dominación y explotación en estas sociedades", APPLE, M. W., "Educación y poder", Paidós, Barcelona, 1987, op. cit. p. 25.

${ }^{33}$ APPLE, M. W., "Educación...", op. cit. p. 56. 
asignación o distribución ${ }^{34}$ - , encuentra el punto en común de ambas, y éste no es otro que el de otorgar un determinado papel a la escuela como el dirigido a la reproducción de la desigualdad, necesitando dar el enfoque no sólo productivo de esta desigualdad, sino distributivo, produciendo también capital humano. ${ }^{35}$

\section{CONSIDERACIONES SOBRE LA RELACIÓN DE LOS DERECHOS DE EDUCACIÓN, TRABAJO Y DESARROLLO.}

No se duda que el derecho a la educación tiene el carácter de social y fundamental, con posibilidad de accionarse ante los tribunales. PRIETO señala: "...el legislador dispone de una amplia discrecionalidad para organizar la enseñanza, pero al final ha de garantizar la escolarización gratuita de todos los niños en los niveles básicos, y esta es sin más una pretensión ante los

\footnotetext{
${ }^{34}$ Explica APPLE: "Que existe relación entre el conocimiento y la economía no es nada nuevo. A menudo se reconoce, aunque no desde los mismos puntos de vista, por parte de marxistas y no marxistas. Por ejemplo, dos aproximaciones recientes - una supuestamente neutra, la otra de izquierdas - son algunas de las respuestas a esta relación, y han dominado nuestro pensamiento sobre la función de la escuela. Aunque básicamente cada una no sea más que un tipo ideal que atenúa las diferencias entre ellas, pueden denominarse teorías del capital humano y de asignación o distribución. La primera afirma que las escuelas son agentes fundamentales en el desarrollo industrial y en la movilidad. Las escuelas maximizarían la distribución del conocimiento técnico y administrativo entre la población. En tanto los estudiantes adquieren este conocimiento, podrán invertir estas destrezas y experiencias adquiridas y progresarán en la escala hacia mejores ocupaciones. Esto les proporcionará niveles de movilidad individual elevados, y también les garantizará la oferta de personas bien preparadas, precisas para la expansión económica. Preparación técnica general, movilidad y crecimiento económico van unidos. Aquí son imprescindibles tanto la planificación consciente de la "fuerza de trabajo" como el estímulo de los currícullums cientificos, técnicos y profesionales en la escuela. Casi como respuesta directa a las ideas de muchos miembros de la escuela del capital humano, los teóricos de la distribución afirman lo contrario. La escuela no está para estimular la movilidad de clase. Es más, actúa como clasificadora. Coloca a los individuos en sus "lugares correctos" dentro de la división jerárquica del trabajo, y distribuye las disposiciones, normas y valores (a través de los currículums ocultos) que requieren los trabajadores para una participación real en su peldaño de la escala laboral. La enseñanza de mensajes segregados (según la posición social), la relativamente escasa importancia de la competencia técnica y una carencia de movilidad entre clases van unidas. Para los teóricos del capital humano, la "apropiación" de conocimientos conduce a un mayor poder personal en el aspecto económico. Para los teóricos de la distribución, la interiorización del conocimiento ocultos - disposiciones, normas y valores - conduce a un poder permanente en manos de los otros.", APPLE, M. W., "Educación...", op. cit. pp. 59 y 60.

${ }^{35}$ Así: "(...) hay una interacción, que es muy compleja, entre el papel de la escuela en la producción de agentes para la división social del trabajo (un papel que los economistas políticos de la educación han reconocido) y la actuación de la escuela como productora de capital cultural."APPLE, M. W., "Educación...", op. cit. pp. 59 y 60.
} 
Tribunales, incluido el Constitucional." ${ }^{36}$ Es decir, la educación es un pilar del progreso y del desarrollo subjetivo y objetivo, para así poder hacer efectivos otros derechos en la edad adulta, como son la libre elección de profesión y la de la igualdad en este terreno y en el del resto de ámbitos. A nivel objetivo, desde el momento en el que en un Estado existen niños trabajando implica un acortamiento de la vida de éstos, y, en consecuencia, una depresión económica mayor de la sociedad en la que se insertan. Por eso, la educación ha de ser uno de los instrumentos vitales a la hora de erradicar el trabajo infantil. Existen muchos estudios sobre cuál es el mejor modelo de educación, pero en todo caso ésta ha de ser una institución democrática que no puede servir para poner en peligro la salud física y mental de la juventud, de forma que no se recibirá una educación adecuada cuando ésta tenga que combinarse con trabajos que perjudiquen la salud de los menores o cuando su contenido se centre exclusivamente en aprendizajes de técnicas de producción durante la minoría de edad. Por lo tanto, no es suficiente con establecer la obligatoriedad de la educación sino que es importante también en qué entorno se está desenvolviendo ésta.

Llegados a este punto, esto es, que la obligatoriedad de la educación tiene su fundamento en el derecho al desarrollo personal y moral, cabría preguntarse lo siguiente: partiendo de este derecho al desarrollo, ¿son los niños los únicos titulares del mismo, o tienen un "derecho de ejercicio preferente" en tal derecho de educación y/o desarrollo?

En un primer momento se puede concluir que la titularidad y ejercicio del derecho a la educación, como fundamento del crecimiento y desarrollo de la personalidad, corresponde sin distinción de ningún tipo a toda persona, no resultando vedado tal derecho a los mayores de edad o adultos. Pero,

\footnotetext{
${ }^{36}$ PRIETO SANCHÍS, L., "Ley, Principios, Derechos", en Cuadernos "Bartolomé de las Casas", Instituto de Derechos Humanos "Bartolomé de las Casas", Universidad Carlos III de Madrid, Dikynson, 1998, op. cit., p. 103. Continúa: "Sin embargo, el Tribunal Constitucional ha interpretado muy cautamente el derecho al trabajo. En su opinión, este derecho presenta dos dimensiones muy distintas: de libertad, tutelada por el artículo 35, y de prestación, que se adscribe, en cambio, al artículo 40.1, que simplemente establece que los poderes públicos "realizarán una política orientada al pleno empleo", Vid. STC 22/1981", PRIETO SANCHÍS, L., "Ley...", op. cit., p. 103.
} 
¿significa esto que existe un mismo interés de protección del derecho a educarse en el caso de los niños que en de los adultos?

Ante esta disyuntiva a que se puede llegar desde la universalización de los derechos, cual es que toda persona tiene derecho a la educación y al desarrollo de su personalidad, es necesario partir de otras premisas que permitan interpretarlos de la manera adecuada para su resolución, y en esto radica, a nuestro entender, uno de los fundamentos de la prohibición del trabajo de los menores.

Y así, es un hecho que la explotación laboral de los menores impide su desarrollo personal y moral, atacando su dignidad; pero no es menos cierto que la explotación laboral misma también impide el desarrollo, el crecimiento personal, y ataca a la dignidad de la persona del trabajador mayor de edad. Esto no ofrece duda, pero si se aplicara la misma regla para mayores que para menores, y la educación fuera el único fundamento del desarrollo y progreso personal y social, podría llegarse incluso a la abolición del trabajo como algo contrario al desarrollo.

Al contrario, que el trabajo es necesario a nivel personal y general es algo innegable, formado parte de la existencia misma de personas y sociedades, de tal manera que trabajo y desarrollo no son, en absoluto, conceptos antagónicos sino complementarios. Pero el trabajo ha de llevarse a cabo en unas condiciones y bajo unas premisas determinadas que hagan que otorgue dignidad a quien lo desempeña y no lo contrario. Esto se consigue, en primer término, cuando se sabe de tal condición, es decir, cuando existe conciencia de trabajador como la cualidad de una persona que libremente asume desempeñar una labor - física, intelectual, etc---- por un determinado número de horas y por lo que recibe determinada retribución justa.

En el caso del trabajo realizado por los niños, la situación de necesidad y vulnerabilidad existente por las necesidades que han de cubrir hace que no tengan otra salida que desempeñar el mismo. Ese vicio en la voluntad determina la conversión, no en trabajo, sino en esclavitud la situación por la que pasan. La voluntariedad es el elemento aparente y la situación de necesidad es el elemento esencial y real. 
En este terreno es donde el instrumento de la educación ha de actuar en su favor. Su fundamental misión ha de centrarse, además de que adquieran los conocimientos que han de considerarse básicos, en la transmisión del conocimiento y asunción del valor de la dignidad de cada persona humana, y en sus relaciones con los demás. La personalidad humana ha de desarrollarse respetando esto, resultando ser la educación uno de los medios más importantes de transmisión del concepto de dignidad humana. ${ }^{37}$

Es por ello que estimamos que ha de diferenciarse el derecho de educación del derecho al desarrollo. El derecho a la educación obligatoria es un derecho a ser ejercido preferentemente por los menores y que tiene como fundamental finalidad la formación de su personalidad, con el contenido básico que hemos señalado. Esta educación obligatoria es un derecho que forma parte e integra un más amplio derecho al desarrollo, es presupuesto imprescindible de éste, pero no el único, requiriéndose, además, para que alcance realidad, el efectivo desarrollo y condiciones de los mayores de edad. El derecho al desarrollo de los mayores de edad estará determinado también por su acceso a la educación y a la cultura, pero no sólo por esto, sino también por sus condiciones de trabajo y de vida.

\footnotetext{
${ }^{37}$ IMBERT opina que: "Aprendizaje, lenguaje: lo que queda por hacer compete sobre todo a la pedagogía..." Según este autor: "los derechos humanos no son algo innato, sino un hábito adquirido: no son "naturales" (más bien lo que caracteriza al hombre frente a otras especies animales es lo inhumano); se aprenden pero no pueden ser impuestos por decreto. El respeto de la dignidad de cada ser humano no puede garantizarse tan sólo mediante prescripciones normativas", IMBERT, P. H., "Los Derechos Humanos en la Actualidad", en "Derechos Humanos y Constitucionalismo ante el Tercer Milenio", Marcial Pons, Madrid, 1.996, op. cit., 84. Según este autor: "Con ello no hacemos más que confirmar que estamos todavía en un período de aprendizaje de la humanidad y recordar que los derechos humanos son tan sólo el lenguaje de la humanización del hombre.", IMBERT, P. H., "Los Derechos Humanos...", op. cit., p. 84. En el mismo sentido, PÉREZ LUÑO: "Sólo la educación en los valores y principios que informan las libertades y las normas constitucionales pueden asegurar su vigencia.", PÉREZ LUÑO, A. E., "Derechos Humanos y Constitucionalismo en la actualidad ¿Continuidad o cambio de paradigma?", en "Derechos Humanos y Constitucionalismo ante el Tercer Milenio", Marcial Pons, Madrid, 1.996, op. cit. p. 45. Concluye posteriormente: "El punto de partida para establecer un paralelismo entre la educación y los derechos humanos estriba en la idea de comunicación; en el presupuesto antropológico de concebir al ser humano como un ser comunicativo. Sin comunicación no puede existir la transmisión de conocimientos entre los hombres, a raíz de toda experiencia cultural y educativa, ni tampoco pueden existir relaciones de cooperación entre los hombres, que son el sustrato de las comunidades libres. La actividad comunicativa tiene como requisito social la existencia libre e indiscriminada de los hombres y como fin la emancipación humana, valores comunes a la educación y a las libertades.", PÉREZ LUÑO, A. E., "Derechos Humanos y..., op. cit., p. 47.
} 
En consecuencia, defendemos la universalidad en la titularidad y ejercicio del derecho al desarrollo y, a la vez y de forma compatible, la especificación en cuanto a este derecho en el caso de los niños, concretándose de manera muy relevante en la educación obligatoria, como un derecho a ser ejercitado preferentemente por ellos.

\section{REFERÊNCIAS}

1.- Libro/Monografía

APPLE, M. W., "Educación y poder", Paidós, Barcelona, 1987.

CIRIGLIANO, G., F., "Filosofía de la educación"”, Humanitas, Buenos Aires, 1972.

CIPOLLA, C., "Educación y desarrollo en occidente", Ariel, Barcelona, 1970.

CARRO IGELMO, A.J., "Historia social del Trabajo", Bosch, Barcelona, 1985.

CUNNINGHAM, H, "Trabajo y explotación infantil: situación en la Inglaterra de los siglos XVII al XX", Ministerio de Trabajo y Seguridad Social, Madrid, 1994.

FREIRE, P, "Pedagogía del oprimido", Siglo Veintinuno de España Editores, Madrid, 2000.

MARX, K., y ENGELS, F., "Manifiesto Comunista y otros escritos políticos", Grijalbo, Barcelona, 1975.

ROUSSEAU, J.J., "El Emilio o de la Educación”, Edaf, Madrid, 1977.

ZOLÁ, E., "Germinal", Austral, Madrid, 2008.

2.- Artículo de revista

CAMPOY CERVERA, I., "La negación de los derechos de los niños en Platón y Aristóteles", Cuadernos "Bartolomé de las Casas", Dykinson, 2006.

PRIETO SANCHÍS, L., "Ley, Principios, Derechos", en Cuadernos "Bartolomé de las Casas", Instituto de Derechos Humanos "Bartolomé de las Casas", Universidad Carlos III de Madrid, Dikynson, 1998.

3.- Trabajo o artículo incluido en libro 
FRÖBEL, F., "El respeto a la libertad de la naturaleza", en "La educación del hombre", en V.V.A.A., "La educación en los tiempos modernos. Tiempos y documentos". Dir. Julio Ruiz Berrio, Actas, Madrid, 1996.

IMBERT, P. H., "Los Derechos Humanos en la Actualidad", en "Derechos Humanos y Constitucionalismo ante el Tercer Milenio", Marcial Pons, Madrid, 1.996.

MARX, K., "Trabajo de jóvenes y niños de ambos sexos", "Instrucciones a los delegados del Consejo Central Provisional", en "La educación en los tiempos modernos. Tiempos y documentos", Director Julio Ruiz Berrio, Actas, Madrid, 1996.

PÉREZ LUÑO, A. E., "Derechos Humanos y Constitucionalismo en la actualidad ¿Continuidad o cambio de paradigma?", en "Derechos Humanos y Constitucionalismo ante el Tercer Milenio", Marcial Pons, Madrid, 1.996.

SANGUINETTI RAYMOND, W., "El derecho del trabajo como categoría histórica", en V.V.A.A., "Mercado y organización del trabajo en España (Siglos XIX y XX)", Grupo Editorial Atril 97, Sevilla, 1998.

SUBIRATS, M., "Sociología de la Educación" en V.V.A.A. "Sociología en España", Comp. Salvador Giner y Luis Moreno, Consejo Superior de Investigaciones Científicas", Madrid, 1990.

\section{4.- Direcciones en la web}

http://www.ilo.org./ipec/Informationresources.

GORDON, P., "Robert Owen (1771-1858)", Perspectivas: revista trimestral de educación comparada (París, UNESCO: Oficina Internacional de Educación), vol. XXIV, № 1-2, 1993, pp. 279-297. http://www.ibe.unesco.org/fileadmin/user_upload/archive/publicarion/ThinkersP df/owens.pdf. 